\title{
ON THE MAXIMUM OF COTANGENT SUMS RELATED TO THE RIEMANN HYPOTHESIS IN RATIONAL NUMBERS IN SHORT INTERVALS
}

\author{
HELMUT MAIER AND MICHAEL TH. RASSIAS
}

\begin{abstract}
Cotangent sums play a significant role in the Nyman-Beurling criterion for the Riemann Hypothesis. Here we investigate the maximum of the values of these cotangent sums over various sets of rational numbers in short intervals.

Key words: Cotangent sums; Estermann's zeta function; Riemann zeta function; Riemann Hypothesis; Kloosterman sums.

2000 Mathematics Subject Classification: 26A12; 11L03; 11M06.
\end{abstract}

\section{INTRODUCTION}

The subject of this paper, the cotangent sums

$$
c_{0}\left(\frac{r}{b}\right):=-\sum_{m=1}^{b-1} \frac{m}{b} \cot \left(\frac{\pi m r}{b}\right)
$$

has been studied by the authors in various papers (cf. [6, [7, 8], 9], [10, 11, [12, 13], 14, 15], 16, [17) and by the second author in his thesis [18. In [18] the author considers moments of $c_{0}(r / b)$ as the variable $r$ ranges over the set

$$
\left\{r:(r, b)=1, A_{0} b \leq r \leq A_{1} b\right\},
$$

where $A_{0}, A_{1}$ are fixed with $1 / 2<A_{0}<A_{1}<1$ and $b$ tends to infinity. He could show that

$$
\frac{1}{\phi(b)} \sum_{\substack{(r, b)=1 \\ A_{0} b \leq r \leq A_{1} b}} c_{0}\left(\frac{r}{b}\right)^{2 k}=H_{k} b^{2 k}(1+o(1)),(b \rightarrow+\infty),
$$

where

$$
\begin{gathered}
H_{k}:=\int_{0}^{1}\left(\frac{g(x)}{\pi}\right)^{2 k} d x, \\
g(x):=\sum_{l \geq 1} \frac{1-2\{l x\}}{l} .
\end{gathered}
$$

The range $1 / 2<A_{0}<A_{1}<1$ was later extended to $0<A_{0}<A_{1}<1$ by S. Bettin in 2].

The cotangent sums $c_{0}(r / b)$ can be associated to the study of the Riemann Hypothesis through its relation with the so-called Vasyunin sum $V$, which is defined

Date: January 5, 2021. 
as follows:

$$
V\left(\frac{r}{b}\right):=\sum_{m=1}^{b-1}\left\{\frac{m r}{b}\right\} \cot \left(\frac{\pi m r}{b}\right),
$$

where $\{u\}:=u-\lfloor u\rfloor, u \in \mathbb{R}$.

It can be shown that

$$
V\left(\frac{r}{b}\right)=-c_{0}\left(\frac{\bar{r}}{b}\right)
$$

where $\bar{r}$ is such that $\bar{r} r \equiv 1(\bmod b)$.

The Vasyunin sum is itself associated to the study of the Riemann Hypothesis through the following identity (see [1], [3]):

$$
\begin{aligned}
& \frac{1}{2 \pi(r b)^{1 / 2}} \int_{-\infty}^{+\infty}\left|\zeta\left(\frac{1}{2}+i t\right)\right|^{2}\left(\frac{r}{b}\right)^{i t} \frac{d t}{\frac{1}{4}+t^{2}} \\
& =\frac{\log 2 \pi-\gamma}{2}\left(\frac{1}{r}+\frac{1}{b}\right)+\frac{b-r}{2 r b} \log \frac{r}{b}-\frac{\pi}{2 r b}\left(V\left(\frac{r}{b}\right)+V\left(\frac{b}{r}\right)\right) .
\end{aligned}
$$

According to this approach initiated by Nyman and Beurling, the Riemann Hypothesis is true if and only if

$$
\lim _{N \rightarrow+\infty} d_{N}=0
$$

where

$$
d_{N}^{2}:=\inf _{D_{N}} \frac{1}{2 \pi} \int_{-\infty}^{+\infty}\left|1-\zeta\left(\frac{1}{2}+i t\right) D_{N}\left(\frac{1}{2}+i t\right)\right|^{2} \frac{d t}{\frac{1}{4}+t^{2}}
$$

and the infimum is taken over all Dirichlet polynomials

$$
D_{N}(s)=\sum_{n=1}^{N} \frac{a_{n}}{n^{s}}
$$

In the paper [11] the authors investigate the maximum of $\left|c_{0}(r / b)\right|$ for fixed large $b$ and $r$ lying in a short interval $\left[A_{0} b,\left(A_{0}+\Delta\right) b\right], 0<A_{0}<1, \Delta=b^{-C}, 0<C<1 / 2$ fixed. We recall the following definitions and results from [11]:

Definition 1.1. Let $0<A_{0}<1,0<C<1 / 2$. For $b \in \mathbb{N}$ we set

$$
\Delta:=\Delta(b, C)=b^{-C} \text {. }
$$

We set

$$
M\left(b, C, A_{0}\right):=\max _{A_{0} b \leq r \leq\left(A_{0}+\Delta\right) b}\left|c_{0}\left(\frac{r}{b}\right)\right| .
$$

Theorem 1.2. (Theorem 1.2 of [1])

With Definition 1.1 let $D$ satisfy $0<D<\frac{1}{2}-C$. Then we have for sufficiently large b:

$$
M\left(b, C, A_{0}\right) \geq \frac{D}{\pi} b \log b .
$$

In this paper we modify this result in two directions:

I) We restrict the numerator $r$ in [11] to the sequence of prime numbers. We shall prove:

Theorem 1.3. Let $q$ be prime, $C, D>0, C+D<1 / 32$. Let $\Delta$ be defined as in Definition 1.1.

$$
M_{p}\left(q, C, A_{0}\right):=\max _{\substack{A_{0} q \leq p \leq\left(A_{0}+\Delta\right) q \\ p \text { prime } \\ 2}}\left|c_{0}\left(\frac{p}{q}\right)\right| .
$$


Then we have for sufficiently large q:

$$
M_{p}\left(q, C, A_{0}\right) \geq \frac{D}{\pi} q \log q .
$$

II) We consider fractions $r / b$ simultaneously varying the numerator $r$ and the denominator $b$.

Definition 1.4. For $\alpha \in(0,1), \Delta>0, B \in \mathbb{N}$ we define

$$
\begin{gathered}
\mathcal{R}(\alpha, \Delta, B):=\left\{\frac{r}{b}:\left|\alpha-\frac{r}{b}\right|<\Delta,(r, b)=1, B \leq b \leq 2 B\right\}, \\
M_{s}(\alpha, \Delta, B):=\max _{\frac{r}{b} \in \mathcal{R}(\alpha, \Delta, B)}\left|c_{0}\left(\frac{r}{b}\right)\right| .
\end{gathered}
$$

We shall prove:

Theorem 1.5. Let $\alpha \in(0,1), 0<C, D<1, C+D<3 / 4$. Then we have for $B$ sufficiently large:

$$
M_{s}\left(\alpha, B^{-C}, B\right) \geq \frac{D}{\pi} B \log B .
$$

Basic for the proof of the result of the paper [11 as well as for the proofs of the results of the present paper is the relation of $c_{0}(r / b)$ to the Estermann zeta function $E\left(s, \frac{r}{b}, \alpha\right)$ and the closely related function $D_{\sin }(s, x)$. We give the following definition and lemma.

Definition 1.6. Let $\operatorname{Re} s>\operatorname{Re} \alpha+1, b \geq 1,(r, b)=1$ and

$$
\sigma_{\alpha}(n):=\sum_{d \mid n} d^{\alpha} .
$$

The Estermann zeta function is defined by

$$
E\left(s, \frac{r}{b}, \alpha\right):=\sum_{n \geq 1} \frac{\sigma_{\alpha}(n) \exp (2 \pi i n r / b)}{n^{s}} .
$$

For $x \in \mathbb{R}$, Res $>1$, we set

$$
D_{\sin }(s, x):=\sum_{n \geq 1} \frac{d(n) \sin (2 \pi n x)}{n^{s}} .
$$

Lemma 1.7.

$$
c_{0}\left(\frac{r}{b}\right)=\frac{1}{2} D_{\sin }\left(0, \frac{r}{b}\right)=2 b \pi^{-2} D_{\sin }\left(1, \frac{\bar{r}}{b}\right) .
$$

Proof. This is Lemma 2.6 of [1].

From Lemma 1.7 it becomes clear, that a crucial step in the proofs has to be the simultaneous localization of the fractions $r / b$ and $\bar{r} / b$. After confining $r / b$ and $\bar{r} / b$ to certain intervals and approximating the characteristic functions of these intervals by Fourier series, this leads to the problem of estimating certain exponential sums. In [11] Kloosterman sums with the fixed denominator $b$ are estimated by a result due to A. Weil. If the numerators $r$ are restricted to special subsets of the integers, like prime numbers, other exponential sums - in the present paper exponential sums in finite fields - must be considered. We apply estimates due to Fouvry and Michel [5].

If both numerators and denominators are variable, sums of Kloosterman sums have 
to be considered. We shall apply results based on the Spectral Theory of Automorphic Forms due to Deshouillers and Iwaniec [4].

In Section 4 we collect all definitions and results on these exponential sums, needed for the proofs of Theorems 1.3 and 1.5

\section{Preliminary Lemmas}

In the following lemma we give a relation between the value $D_{\sin }(1, x)$ and the continued fraction expansion of $x$.

Lemma 2.1. Let $x=\left\langle a_{0} ; a_{1}, a_{2}, \ldots\right\rangle$ be the continued fraction expansion of $x \in \mathbb{R}$. Moreover, let $u_{r} / v_{r}$ be the $r$-th partial quotient of $x$. Then

$$
D_{\text {sin }}(1, x)=-\frac{\pi^{2}}{2} \sum_{l \geq 1} \frac{(-1)^{l}}{v_{l}}\left(\left(\frac{1}{\pi v_{l}}\right)+\psi\left(\frac{v_{l-1}}{v_{l}}\right)\right),
$$

whenever either of the two series (1.3), (2.1) is convergent.

If $x=\left\langle a_{0} ; a_{1}, a_{2}, \ldots, a_{r}\right\rangle$ is a rational number then the range of summation of the series on the right is to be interpreted to be $1 \leq l \leq r$. Here $\psi$ is an analytic function satisfying

$$
\psi(x)=-\frac{\log (2 \pi x)-\gamma}{\pi x}+O(\log x),(x \rightarrow 0) .
$$

Proof. This is Lemma 2.5 of [1].

Lemma 2.2. Let $\epsilon>0, b \geq b(\epsilon),(r, b)=1,0<r<b$. Let

$$
\frac{r}{b}=\left\langle 0 ; w_{1}, \ldots, w_{s}\right\rangle
$$

be the continued fraction expansion of $r / b$ with partial fractions $u_{i} / v_{i}$. Then there are at most 3 values of $l$ for which

$$
\frac{1}{v_{l}} \psi\left(\frac{v_{l-1}}{v_{l}}\right) \geq \log \log b
$$

and at most one value of $l$, for which

$$
\frac{1}{v_{l}} \psi\left(\frac{v_{l-1}}{v_{l}}\right) \geq \epsilon \log b .
$$

Proof. This is Lemma 2.14 of [11].

\section{Fourier Analysis}

Definition 3.1. For $\beta \in \mathbb{R}, v \geq 0, \Delta>0$, let the functions $\chi_{1}, \chi_{2}$ be defined by

$$
\chi_{1}(u, v):= \begin{cases}1, & \text { if } \beta+v<u \leq \beta+\Delta-v \\ 0, & \text { otherwise }\end{cases}
$$

and

$$
\chi_{2}(u):=\Delta^{-1} \int_{0}^{\Delta} \chi_{1}(u, v) d v .
$$

Lemma 3.2. We have

$$
\chi_{2}(u)=\sum_{n=-\infty}^{\infty} a(n) e(n u)
$$


where $a(0)=\Delta / 2$ and

$$
a(n)= \begin{cases}O(\Delta), & \text { if }|n| \leq \Delta^{-1} \\ O\left(\Delta^{-1} n^{-2}\right), & \text { if }|n|>\Delta^{-1} .\end{cases}
$$

Proof. This is Lemma 2.10 of [11].

Definition 3.3. For $\gamma>0, v \geq 0$, let

$$
\chi_{3}(u, v):= \begin{cases}1, & \text { if }-\gamma+v<u<\gamma \\ 0, & \text { otherwise }\end{cases}
$$

and

$$
\chi_{4}(u):=\gamma^{-1} \int_{0}^{\gamma} \chi_{3}(u, v) d v .
$$

Lemma 3.4. We have

$$
\chi_{4}(u)=\sum_{n=-\infty}^{+\infty} c(n) e(n u)
$$

where $c(0)=\gamma$ and

$$
c(n)= \begin{cases}O(\gamma), & \text { if }|n| \leq \gamma^{-1} \\ O\left(\gamma^{-1} n^{-2}\right), & \text { if }|n|>\gamma^{-1} .\end{cases}
$$

Proof. This is Lemma 2.22 of [11].

\section{Exponential Sums}

Definition 4.1. Let $b \in \mathbb{N}, m, n \in \mathbb{Z}$. The Kloosterman sum $K(m, n, b)$ is defined by

$$
K(m, n, b):=\sum_{\substack{r=1 \\(r, b)=1}}^{b-1} e\left(\frac{m r+n \bar{r}}{b}\right) .
$$

For $m=0$ (resp. $n=0)$ we obtain the Ramanujan sums $K(0, n, b)($ resp. $K(m, 0, b))$.

Lemma 4.2. We have the bounds

$$
|K(m, n, b)| \leq d(b)(m, n, b)^{1 / 2} b^{1 / 2}
$$

and

$$
|K(0, n, b)| \leq(n, b) .
$$

Proof. The result (4.1) is due to Weil (cf. [19]). The result (4.2) is elementary.

The next result has not been used in previous papers of the authors. It is due to Deshouillers and Iwaniec and is related to the Spectral Theory of Automorphic Forms.

Lemma 4.3. For positive real numbers $T, M, N, \epsilon$ and complex sequences $\vec{a}=\left(a_{m}\right)_{m \in \mathbb{N}}$, $\vec{b}=\left(b_{n}\right)_{n \in \mathbb{N}}$ one has

$$
\begin{gathered}
\sum_{M<m \leq 2 M} a_{m} \sum_{N<n \leq 2 N} b_{n} \sum_{b \leq\left(\frac{m n}{M N}\right)^{\frac{1}{2}} T} \frac{1}{b} K(m, \pm n, b) \\
\ll T^{\epsilon}\left\{(M N)^{1 / 2}+(T M N)^{1 / 6}\right\}\left\|a_{M}\right\|_{2}\left\|b_{N}\right\|_{2} . \\
5
\end{gathered}
$$


The constant implied in $\ll$ depends on $\epsilon$ alone. Here $\left\|c_{N}\right\|_{2}$ is defined by

$$
\left\|c_{N}\right\|_{2}:=\left(\sum_{N<n \leq 2 N}\left|c_{N}\right|^{2}\right)^{1 / 2} .
$$

Proof. This is formula (1.47) from the Corollary to Theorem 8 in [4].

Definition 4.4. Let $\mathbb{F}_{q}$ be the finite field with $q$ elements and let $\psi$ be a non-trivial additive character over $\mathbb{F}_{q}, f$ a rational function of the form

$$
f(x)=\frac{P(x)}{Q(x)},
$$

$P$ and $Q$ relatively prime, monic non-constant polynomials,

$$
S(f ; q, x):=\sum_{p \leq x} \psi(f(p)),
$$

( $p$ denotes the $p$-fold sum of the element 1 in $\mathbb{F}_{q}$ ).

Lemma 4.5. With conditions from Definition 4.4 we have:

$$
S(f ; q, x) \ll q^{3 / 16+\epsilon} x^{25 / 32} .
$$

The implied constant depends only on $\epsilon$ and the degrees of $P$ and $Q$.

Proof. This is due to Fouvry and Michel [5].

\section{Proof of Theorem 1.3}

Definition 5.1. Let $\Delta=b^{-C}$ as in Definition 1.1 and let $\Omega>0$. Let $q$ be a prime number. We set

$$
N(q, \Delta, \Omega):=\left\{p \text { prime : } A_{0} q \leq p \leq\left(A_{0}+\Delta\right) q,|\bar{p}| \leq \Omega\right\} .
$$

Definition 5.2. Let $S(f ; q, x)$ be as in Definition 4.4. Let $E(m, n, q):=S(f ; q, q)$ with $f(p):=m p+\frac{n}{p}, \psi(u):=e(u / q)$.

Lemma 5.3. We have

$$
E(m, n, q) \ll q^{31 / 32+\epsilon}
$$

for all $\epsilon>0$.

Proof. This follows from Lemma 4.5 with $x=q$.

Lemma 5.4. We have

$$
N(q, \Delta, \Omega)>0
$$

for q sufficiently large.

Proof. By Definitions 3.1, 3.3, 5.1 and Lemmas 3.2, 3.4, 5.3 we have

$$
\begin{aligned}
N(q, \Delta, \Omega) & \geq \phi(q) a(0) c(0)+\sum_{m, n=-\infty}^{\infty} a(m) c(n)|E(m, n, q)| \\
& \geq \phi(q) q^{-(C+D+\epsilon)}+O\left(q^{31 / 32+\epsilon}\right),
\end{aligned}
$$

which proves the result. 
We may now conclude the result of Theorem 1.3 By Lemma 5.4 there is at least one prime $p \in\left[A_{0} q,\left(A_{0}+\Delta\right) q\right]$, such that $\frac{\bar{p}}{q} \in(0, \Omega)$.

By Lemma 2.1 we have:

$$
c_{0}\left(\frac{p}{q}\right)=-q \sum_{l \geq 1} \frac{(-1)^{l}}{v_{l}}\left(\frac{1}{\pi v_{l}}+\psi\left(\frac{v_{l-1}}{v_{l}}\right)\right) .
$$

Let $\left(u_{i} / v_{i}\right)_{i=1}^{s}$ be the sequence of partial fractions of $\frac{\bar{p}}{q}$. From

$$
\Omega \geq \frac{\bar{p}}{q} \geq \frac{1}{v_{1}+1}
$$

we obtain $v_{1}+1 \geq \Omega^{-1}$.

By Lemma 2.2 we have

$$
\sum_{l>1}\left(\frac{1}{\pi v_{l}}+\psi\left(\frac{v_{l-1}}{v_{l}}\right)\right)<2 \epsilon \log q, \text { for } q \geq q_{0}(\epsilon) .
$$

Therefore,

This proves Theorem 1.3

$$
\left|D_{\sin }\left(0, \frac{p}{q}\right)\right| \geq \frac{1}{\pi} \log \left(\Omega^{-1}(1+o(1)), \quad(q \rightarrow \infty) .\right.
$$

\section{Proof of Theorem 1.5}

Definition 6.1. Let $\alpha \in(0,1), \Delta>0, \Omega>0$. We set

$$
N(\alpha, \Delta, \Omega):=\#\left\{(b, r): \alpha \leq \frac{r}{b} \leq \alpha+\Delta,(r, b)=1,|\bar{r}| \leq \Omega b, B<b \leq 2 B\right\} .
$$

Lemma 6.2. Let $0<C, D<1, C+D<3 / 4$. Then we have:

$$
N(\alpha, \Delta, \Omega)>0 \text {. }
$$

Proof. By Definition 6.1, Lemmas 3.2, 3.4 we have with a positive constant $c^{*}>0$ :

$$
N(\alpha, \Delta, \Omega) \geq c^{*} B^{2} a(0) c(0)+\sum_{\substack{(m, n)=-\infty \\(m, n) \neq(0,0)}}^{\infty} a(m) c(n) \sum_{B<b \leq 2 B} K(m, n, b)
$$

It suffices to treat only the terms with $m>0, n>0$. We partition the sum into subsums:

$$
\Sigma_{M, N}:=\sum_{M<m \leq 2 M} a_{m} \sum_{N<n \leq 2 N} c_{n} \sum_{B<b \leq 2 B} K(m, n, b) .
$$

We obtain $\Sigma_{M, N}$ by partial summation from

$$
\Sigma_{M, N, u}:=\sum_{M<m \leq 2 M} a_{m} \sum_{N<n \leq 2 N} c_{n} \sum_{1 \leq b \leq u} \frac{1}{b} K(m, n, b) .
$$

We choose integers $\mu=\mu(M)$ and $\nu=\nu(N)$ to be determined later and partition the interval $(M, 2 M]$ into $O(\mu)$ subintervals $I_{k}:=\left(m_{k}, m_{k+1}\right]$ of lengths $\left|I_{k}\right|$ with

$$
\frac{1}{2} \mu^{-1} M<\left|I_{k}\right| \leq 2 \mu^{-1} M, \quad 1 \leq k \leq k_{0}(M)
$$

and the interval $(N, 2 N]$ into $O(v)$ subintervals $J_{l}:=\left(n_{l}, n_{l+1}\right]$ of lengths $\left|J_{l}\right|$ with

$$
\frac{1}{2} \nu^{-1} N<\left|J_{l}\right| \leq 2 \nu^{-1} N, \quad 1 \leq l \leq l_{0}(N)
$$


We have

$$
\Sigma_{M, N, u}=\sum_{1 \leq k \leq k^{*}(M)} \sum_{1 \leq l \leq l^{*}(N)} \Sigma_{M, N, u}^{(k, l)}
$$

where

$$
\Sigma_{M, N, u}^{(k, l)}=\sum_{m \in I_{k}} a_{m} \sum_{n \in J_{l}} c_{n} \sum_{1 \leq b \leq u} \frac{1}{b} K(m, n, b) .
$$

We now partition the sums $\Sigma_{M, N, u}^{(k, l)}$ into two subsums. For this purpose we define $T=T(k, l, u)$ by

$$
T\left(\frac{m_{k} n_{l}}{M N}\right)^{\frac{1}{2}}=u
$$

and set

$$
\Sigma_{M, N, u}^{(k, l)}:=\Sigma_{M, N, u}^{(k, l, 1)}-\Sigma_{M, N, u}^{(k, l, 2)},
$$

where

$$
\begin{aligned}
& \Sigma_{M, N, u}^{(k, l, 1)}:=\sum_{m \in I_{k}} a_{m} \sum_{n \in J_{l}} c_{n} \sum_{\substack{1 \leq b \leq T\left(\frac{m n}{M N}\right)^{\frac{1}{2}} \\
b}} \frac{1}{b} K(m, n, b) \\
& \Sigma_{M, N, u}^{(k, l, 2)}:=\sum_{m \in I_{k}} a_{m} \sum_{n \in J_{l}} c_{n} \sum_{u \leq b \leq T\left(\frac{m n}{M N}\right)^{\frac{1}{2}}} \frac{1}{b} K(m, n, b)
\end{aligned}
$$

We write $M=B^{C} 2^{\kappa}, N=B^{D} 2^{\lambda}$, with $\kappa, \lambda \in \mathbb{Z}$.

We apply Lemma 4.3 with the sequences $\left(a_{m, k}\right),\left(b_{n, l}\right)$ in place of $\vec{a}$ and $\vec{b}$, which we denote by

$$
\begin{aligned}
a_{m, k} & := \begin{cases}a_{m}, & \text { if } m \in I_{k} \\
0, & \text { otherwise },\end{cases} \\
b_{n, l} & := \begin{cases}b_{n}, & \text { if } n \in J_{l} \\
0, & \text { otherwise } .\end{cases}
\end{aligned}
$$

We obtain by Lemmas 4.3 , 3.2, 3.4.

$$
\begin{gathered}
\left\|a_{m, k}\right\|_{2}= \begin{cases}O\left(\mu^{-\frac{1}{2}} B^{-\frac{C}{2}} 2^{\frac{\kappa}{2}}\right), & \text { if } \kappa \leq 0 \\
O\left(\mu^{-\frac{1}{2}} B^{-\frac{C}{2}} 2^{-\kappa}\right), & \text { if } \kappa>0,\end{cases} \\
\left\|b_{n, l}\right\|_{2}= \begin{cases}O\left(\nu^{-\frac{1}{2}} B^{-\frac{D}{2}} 2^{\frac{\lambda}{2}}\right), & \text { if } \lambda \leq 0 \\
O\left(\nu^{-\frac{1}{2}} B^{-\frac{D}{2}} 2^{-\lambda}\right), & \text { if } \lambda>0 .\end{cases}
\end{gathered}
$$

We now estimate $\Sigma_{M, N, u}^{(k, l, 2)}$. We let

$$
\mathcal{J}_{(k, l, u)}^{M, N}:=\left\{(m, n, b): m \in I_{k}, n \in J_{l}, u<b \leq T(m, n, u)\right\} .
$$

We have

$$
\left|\mathcal{J}_{(k, l, u)}^{M, N}\right| \ll 2^{\kappa+\lambda} B^{1+C+D}\left(\mu^{-1}+\nu^{-1}\right) \mu^{-1} \nu^{-1} .
$$

For $(m, n, b) \in \mathcal{J}_{(k, l, u)}^{M, N}$ we estimate the sums $K(m, n, b)$ individually by the use of Lemma 4.2 and obtain by (6.2), (6.3), (6.4) and (6.5):

$$
\sum_{\substack{M, N \\ k, l}} \sum_{(m, n, b) \in \mathcal{J}(M, N, k, l, u)} a_{m, k} c_{n, l} K(m, n, b)=O\left(B^{\frac{3}{2}+\epsilon}\left(\mu^{-1}+\nu^{-1}\right)\right) .
$$


From (6.2), (6.3), (6.6) we finally get:

$$
N(\alpha, \Delta, \Omega)=c^{*} B^{2} a(0) c(0)+O\left(B \mu^{\frac{1}{2}} \nu^{\frac{1}{2}}\right)+O\left(B^{\frac{3}{2}}\left(\mu^{-1}+\nu^{-1}\right)\right) .
$$

We have

$$
a(0) c(0) \geq B^{-C-D+\epsilon} .
$$

We choose $\mu=\nu=B^{\frac{1}{4}}$ and obtain the proof of Lemma 6.2 for the case $C>1 / 4$. For the case $C \leq 1 / 4$ we only partition the interval $(N, 2 N]$ and sum over the contributions of the different values of $m$. Instead of $T=T(k, l, u)$, defined by

$$
T\left(\frac{m_{k} u_{l}}{M N}\right)^{\frac{1}{2}}=u
$$

we define $T=T(l, u)$ by

$$
T\left(\frac{n_{l}}{N}\right)^{\frac{1}{2}}=u
$$

$\mathcal{J}_{(k, l, u)}^{M, N}$ in (6.4) is replaced by

$$
\mathcal{J}_{(l, u)}^{N}:=\left\{(n, b): n \in J_{l}, u<b \leq T(l, u)\right\} .
$$

We again estimate the Kloosterman sums for $(n, b) \in \mathcal{J}_{(l, u)}^{N}$ individually by Lemma 4.2 and for the other pairs $(n, b)$ by Lemma 4.3 This proves Lemma 6.2 also for $C \leq 1 / 4$. The case $D \leq 1 / 4$ is analogous.

Thus the proof of Lemma 6.2 is finished.

We may now conclude the proof of Theorem 1.5

By Lemma 6.2 there is at least one pair $(b, r)$, such that $(r, b)=1$,

$$
\alpha \leq \frac{1}{b}<\alpha+\Delta, \quad\left|\frac{\bar{r}}{b}\right| \leq \Omega, \quad B<b \leq 2 B .
$$

Let $\left(u_{i} / v_{i}\right)_{i=1}^{s}$ be the sequence of partial fractions of $\bar{r} / b$. From

$$
\Omega \geq \frac{\bar{r}}{b} \geq \frac{1}{v_{1}+1}
$$

we obtain $v_{1}+1 \geq \Omega^{-1}$.

By Lemma 2.1 we have:

$$
c_{0}\left(\frac{r}{b}\right)=-b \sum_{l \geq 1} \frac{(-1)^{l}}{v_{l}}\left(\frac{1}{\pi v_{l}}+\psi\left(\frac{v_{l-1}}{v_{l}}\right)\right) .
$$

By Lemma 2.2 we have

$$
\sum_{l \geq 1}\left(\frac{1}{\pi v_{l}}+\psi\left(\frac{v_{l-1}}{v_{l}}\right)\right)<2 \epsilon \log B, \text { for } B \geq B_{0}(\epsilon) .
$$

Therefore,

$$
\left|D_{\sin }\left(0, \frac{r}{b}\right)\right| \geq \frac{1}{\pi} \log \left(\Omega^{-1}(1+o(1)), \quad(B \rightarrow \infty) .\right.
$$

This proves Theorem 1.5. 


\section{REFERENCES}

[1] S. Bettin, A generalization of Rademacher's reciprocity law, Acta Arithmetica, 159(4)(2013), 363-374.

[2] S. Bettin, On the distribution of a cotangent sum, Int. Math. Res. Notices (2015), doi: 10.1093/imrn/rnv036

[3] S. Bettin and B. Conrey, Period functions and cotangent sums, Algebra \& Number Theory $7(1)(2013), 215-242$.

[4] J. -M. Deshouillers and H. Iwaniec, Kloosterman sums and Fourier coefficients of cusp forms, Invent. Math., 70(3)(1982/83), 219-288.

[5] E. Fouvry and Ph. Michel, Sur certaines sommes d'exponentielles sur les nombres premiers, Annales Scientifiques de l'Ecole Normale Supérieure , 4e serie, T. 31, (1998) 93-130.

[6] H. Maier and M. Th. Rassias, Generalizations of a cotangent sum associated to the Estermann zeta function, Communications in Contemporary Mathematics, 18(1)(2016), doi: 10.1142/S0219199715500789.

[7] H. Maier and M. Th. Rassias, The order of magnitude for moments for certain cotangent sums, Journal of Mathematical Analysis and Applications, 429(1)(2015), 576-590.

[8] H. Maier and M. Th. Rassias, The rate of growth of moments of certain cotangent sums, Aequationes Mathematicae, 2015, 90(3)(2016), 581 - 595.

[9] H. Maier and M. Th. Rassias, Asymptotics for moments of certain cotangent sums, Houston Journal of Mathematics, 43(1)(2017), 207-222.

[10] H. Maier and M. Th. Rassias, Asymptotics for moments of certain cotangent sums for arbitrary exponents, Houston Journal of Mathematics, 43(4)(2017), 1235-1249.

[11] H. Maier and M. Th. Rassias, The maximum of cotangent sums related to Estermann's zeta function in rational numbers in short intervals, Applicable Analysis and Discrete Mathematics, 11(2017), 166-176.

[12] H. Maier and M. Th. Rassias, On the size of an expression in the Nyman-Beurling-BaezDuarte criterion for the Riemann Hypothesis, Canadian Mathematical Bulletin, 61(3)(2018), 622-627.

[13] H. Maier and M. Th. Rassias, Estimates of sums related to the Nyman-Beurling criterion for the Riemann Hypothesis, Journal of Number Theory, 188(2018), 96-120.

[14] H. Maier and M. Th. Rassias, Explicit estimates of sums related to the Nyman-Beurling criterion for the Riemann Hypothesis, Journal of Functional Analysis, 276(2019), 3832-3857.

[15] H. Maier and M. Th. Rassias, Distribution of a cotangent sum related to the NymanBeurling criterion for the Riemann Hypothesis, Applied Mathematics and Computation, 363(15)(2019), https://doi.org/10.1016/j.amc.2019.124589.

[16] H. Maier and M. Th. Rassias, Cotangent sums related to the Riemann Hypothesis for various shifts of the argument, Canadian Mathematical Bulletin, 63(3)(2020), 522-535.

[17] H. Maier, M. Th. Rassias and A. Raigorodskii, The maximum of cotangent sums related to the Nyman-Beurling criterion for the Riemann Hypothesis, In: Trigonometric Sums and their Applications, Springer, 2020, 149-158.

[18] M. Th. Rassias, Analytic investigation of cotangent sums related to the Riemann zeta function, Doctoral Dissertation, ETH-Zürich, Switzerland, 2014.

[19] A. Weil, Sur les courbes algébriques et les variétés qui s'en déduisent, Paris, Hermann (1948).

Department of Mathematics, University of Ulm, Helmholtzstrasse 18, 89081 Ulm, Germany.

Email address: helmut.maier@uni-ulm.de

Institute of Mathematics, University of Zurich, CH-8057, Zurich, Switzerland \& Moscow Institute of Physics and Technology 141700 Dolgoprudny, Institutskiy per, D. 9, Russia \& Institute for Advanced Study, Program in Interdisciplinary Studies, 1 Einstein Dr, Princeton, NJ 08540, USA.

Email address: michail.rassias@math.uzh.ch, michailrassias@math.princeton.edu 